\title{
Best practice in access arrangements made for England's General Certificates of Secondary Education (GCSEs): Where are we 10 years on?
}

\author{
Kevin Woods Abi James Amanda Hipkiss
}

Abstract

Ten years after an original survey, the present paper reports findings from a 2017 survey of secondary school staff involved in school-based management of GCSE examination access arrangements. 263 respondents, including specialist assessors and special educational needs co-ordinators (SENCos), explained their views on the manageability and fairness of processes for GCSE examination access arrangements. Whilst perceived fairness of GCSE access arrangements has increased, a majority of respondents does not consider current processes to be manageable at school level or equally fair to students. However, almost two thirds of respondents would support some extension of access arrangements on the basis of student need or use of technological assistance. The researchers recommend: promotion of partnership between the qualifications regulator, awarding bodies and schools; enhanced shared understanding of the purpose, place and limitations of access arrangements; and use of a school-based protocol to manage roles and resource requirements for management access arrangements. 


\section{Introduction}

In 2007, this journal published a research article entitled 'Access to General Certificate of Secondary Education (GCSE) examinations for students with special educational needs: what is best practice?' (Woods, 2007). The aim of that study, which surveyed secondary specialist teachers, was to shed light upon the perceived appropriateness and manageability of provisions and processes for making GCSE examination access arrangements. The survey was undertaken in response to awarding bodies' recently stated aspiration to develop access arrangements regulations in light of best practice, but in a context which included no mechanisms for regular and systematic collection of stakeholder feedback. However, Woods (2007) concluded that 'the general pattern of survey responses suggests that, for teacher stakeholders, the system for access arrangements in GCSE examinations does not hold up well in relation to a best practice ideal' (p.93). Citing similar programmes in the US, Woods (2007) recommended more extensive empirical research into the effectiveness and feasibility of a range of examination access arrangements, alongside evaluation of notions about feasibility and fairness from employers and students, as well as teachers.

Since that time, GCSE examinations have continued to be highly significant to teachers and school leaders as a proxy quality measure for accountability purposes (DeLuca \& Johnson, 2017; Isaacs, 2010). And for students too, the GCSE has continued to be seen as instrumental in their planned transitions to further education, employment and training (Flitcroft, Woods \& Putwain, 2017; Stobart, 2008; Woods, Parkinson \& Lewis, 2010). However, at the same time there have been significant changes in the GCSE examination regime with reduction of coursework, emphasis on terminal examinations, and the ending of controlled assessments, all of which may have a bearing upon the assessment needs of students with disabilities or special educational needs (Department for Education (DfE), 2014a). In addition, focuses upon 'Progress 8 ' ${ }^{i}$ and upon the English Baccalaureate have extended the influence of the GCSE award across the school-leaving age population (DfE, 2015a).

Through technological advances, there have been significant developments of the range of available special provisions incorporated to access arrangements including the introduction of reading pens, computer readers and speech recognition technology (Joint Council for Qualifications (JCQ), 2017). And for specialist teachers, the process for the management and recording of GCSE access arrangements changed significantly in 2008 with the introduction of the 'Access Arrangements Online' system (JCQ, 2008). Furthermore, the DfE has required since 2013 that 
special educational needs co-ordinators (SENCos) manage GCSE access arrangements applications in school, and the JCQ has introduced more stringent requirements for the qualification of specialist assessors.

Another major influence has been the passing of the Equality Act 2010, which requires reasonable adjustments to be made for any disabled person, defined as a person with 'physical or mental impairment and the impairment has a substantial and long-term adverse effect on his or her ability to carry out normal day-to-day activities' (HM Government, 2010, p.4). The Equality Act 2010 (HM Government, 2010) has become central to the aims and guidance framework governing the management of GCSE access arrangements (Ofqual, 2017; JCQ, 2017), though the guidance continues to include within its scope those examination candidates with special educational needs (SEN), which is defined separately and is governed through the Children and Families Act 2014 (DfE, 2014b). Essentially, the notion of disability relates to a functional difficulty, whereas the definition of special educational need focuses more positively and inclusively upon the benefits of special (i.e. not ordinarily available) educational provision(s) necessary for functional effectiveness (DfE, 2015b). The extent to which the JCQ's (2017) GCSE access arrangements guidance has developed to successfully integrate its statutory obligations under the Equality Act 2010, with its broader aims to accommodate students with SEN, has not been scrutinised or researched (cf. Woods, McCaldin, Hipkiss, Tyrell \& Dawes, submitted). Notably, GCSE achievements of students with SEN or a disability are at a lower level than would be predicted (DfE, 2017).

In Woods' (2007) access arrangements survey, a sizable majority of specialist teachers reported from their experience that they did not consider access arrangements administrative processes to be manageable, or the provisions of access arrangements at that time to be equally fair to all students. $70 \%$ of respondents were of the opinion that, notwithstanding resource constraints, they would be in favour of extending the availability of some access arrangements. Specific issues were highlighted including: cost of directed specialist tests of difficulty, e.g. reading accuracy; staff time needed for administration of access arrangements; unreliability of need identification particularly for 'borderline' students; and inappropriateness of a heavily inscriptive assessment regime which effectively 'creates' student assessment needs. It is not clear whether such issues were subsequently resolved, either actively or as a consequence of other developments of the last ten years, or indeed how the current access arrangements system is perceived as being fit-for-purpose. 
There is, however, some evidence of current concerns about rising levels of the use of access arrangements (Ofqual, 2016), albeit that such concerns neither take account of the rise in whole student population, nor justify an expected level of access arrangement applications in respect of the subject irrelevant demands of each examination. Recent media reports suggested that the system for obtaining access arrangements may be susceptible to manipulation through, for example, parental pressure or resource (Woolcock, 2017), though other reports suggested that increased use of access arrangements reflects improved school processes for identification and provisioning (Bateman, 2017). Furthermore, from evidence collated from posts on the SENCo online forum ('SENCo Forum'), Hipkiss and Robertson (2016) presented to The Office of Qualifications and Examinations Regulation (Ofqual) and the Department for Education (DfE) 22 points of concern relating to: the complexity and resource demands of administration processes for GCSE access arrangements; lack of stakeholder consultation and transparency; and lack of candidate perspective within the processes of candidate assessment needs.

Against this background, the aim of the present study is to systematically review the perspectives of secondary school staff with a significant involvement in school-based management of GCSE access arrangements, including SENCos and specialist assessors, to evaluate the extent to which current provisions and processes for GCSE access arrangements may be seen to meet a best practice ideal. A secondary aim is to compare current indications to those described by Woods (2007) ten years ago.

\section{The present study}

\section{Design and instrumentation}

The present study employed a questionnaire survey design, incorporating a combination of closed and open questions (Cohen, Manion \& Morrison, 2011). For comparability purposes, the three open questions included in the survey by Woods (2007) (relating to manageability, fairness and scope of GCSE access arrangements) were included within this survey. In addition, in respect of technological advances since the original survey, two further combined closed/ open questions were included relating respectively to the use, and confidence in use, of assistive technologies as provisions within access arrangements, including a specific focus upon the provisions of word processor, computer reader, a reading pen, and speech recognition software. An initial question requested job role information from each participant and a final open question offered respondents the opportunity to comment upon the impact of access arrangements for students in their setting. 
In this paper, only data relating to issues of access arrangements manageability, fairness and scope are reported and considered. Findings relating to the use of assistive technologies within access arrangements are reported elsewhere (James, Hipkiss \& Woods, in preparation).

\section{Sampling and participant recruitment}

The intended population for participation was secondary age phase school staff in England, including SENCos and specialist assessors, with recent experience of working in school management of GCSE access arrangements in England. Sampling was both opportunistic and purposeful (Gilham, 2007). The questionnaire was distributed in October 2017 at six access arrangement management training events (attended by approximately 850 delegates) at locations across England. These events are intended for, and typically attended by, access arrangements assessors, SENCos and examination officers. The questionnaire was posted online in October 2017 for a period of three weeks on the National Association of Special Educational Needs (NASEN) SENCo online forum, 'SENCo Forum', which has over 2,500 members (www.nasen.co.uk/sencoforum). Finally, the questionnaire was emailed to relevant professional contacts known to each of the three researchers.

\section{Data analysis}

263 responses were received with missing data levels at between 2-5\% across questionnaire items. For efficient management and filtering, all participant data were entered to Microsoft Excel.

Responses to questions relating to manageability and fairness respectively, were categorised a priori as either 'yes', 'ambivalent', 'no', or 'ambiguous'. Job role question responses were coded by category of response; access arrangements impact comments were coded inductively to identify salient themes (Hsieh \& Shannon, 2005). Frequency count tables were compiled for each question category and percentage category responses were calculated; chi-square analyses were carried out for responses to manageability, fairness and scope questions against professional role category. Within each a priori category of response, the content of qualitative data was subjected to directed content analysis to create explanatory subcategories of response (Hsieh \& Shannon, 2005). Within each subcategory, questionnaire text extracts were identified as exemplars for data presentation. For both a priori categories and categories generated from the open question about impact, themes and sub-categories were agreed by all three researchers; where there was an initial difference of interpretation between researchers, this was discussed until agreement was reached. 


\section{Ethical considerations}

Before commencing any data gathering, ethical approval for the research was obtained through the ethical approval committee of a university in the south of England (Reference: 30274). The project adheres to The British Psychological Society (BPS) Code of Human Research Ethics (BPS, 2014). Informed access consent from the training events organisation was obtained. Information about the study and data management (e.g. assured anonymity) was prefaced in the questionnaire, understanding of which was confirmed by each respondent within each returned questionnaire. No specific ethical issues were identified prior to, during or following the research.

\section{Findings}

Table 1 below shows the distribution of main professional roles declared by the 263 respondents, confirming that the respondent group contains both experience relating to the place of access arrangements within a school context, and experience relating to specific student assessments directed by JCQ.

Table 1: Main professional roles of questionnaire respondents $(n=258)$

\begin{tabular}{|l|l|}
\hline Professional role & $\%$ \\
\hline $\begin{array}{l}\text { SENCo/ Access } \\
\text { arrangements manager }\end{array}$ & $43 \%$ \\
\hline Specialist assessor & $47 \%$ \\
\hline Exams officer/ Advisor & $5 \%$ \\
\hline $\begin{array}{l}\text { Support teacher/ } \\
\text { teaching assistant }\end{array}$ & $5 \%$ \\
\hline
\end{tabular}

Table 2 below shows a summary of responses to the questions of manageability, fairness and scope of access arrangements:

Table 2: Distribution of questionnaire responses $(n=263)$

Access arrangements

\begin{tabular}{|l|l|l|l|l|}
\hline & Yes & Ambivalent & No & Ambiguous or no response \\
\hline
\end{tabular}




\begin{tabular}{|l|c|c|c|c|}
\hline Manageable? & $25 \%$ & $24 \%$ & $43 \%$ & $8 \%$ \\
\hline Fair? & $46 \%$ & $30 \%$ & $21 \%$ & $3 \%$ \\
\hline Extension desirable? & $64 \%$ & $10 \%$ & $22 \%$ & $4 \%$ \\
\hline
\end{tabular}

Chi-square analyses of responses to the questions of manageability, fairness and scope of access arrangements show no significant differences across respondent professional role categories.

The current findings indicate relatively little progress over the last decade in stakeholders' perceptions of best practice in relation to the structure and processes for administration of access arrangements for GCSE. In 2007, 20\% of respondents thought the system manageable; this rose to $25 \%$ in 2017 . In $2007,25 \%$ of respondents thought the system to be fair across candidates; this rose to $46 \%$ in 2017. In 2007, $70 \%$ of respondents supported extension of current provisions of access arrangements; this fell to $64 \%$ in 2017.

\section{Manageability of GCSE access arrangements}

Only $25 \%$ of respondents consider the system for administration of GCSE access arrangements to be manageable and $43 \%$ of respondents unequivocally consider it not to be so. Two issues were cited most often:

- Time needed to complete additional JCQ-specified assessments of students, to collect evidence of assessment need ('normal way of working') from subject teachers, and to complete the online application process requirements.

- Incorporation of annual changes in the access arrangements guidance issued by the JCQ was frequently cited as creating demands which were difficult to manage.

Responses from the further education sector consistently highlighted that collection of evidence of need and provision within a new college setting was unfeasible by the required deadline in the first year, particularly as it entails gathering historical information from a student's previous school; similar transition-related issues were raised within three-tier (middle school) education systems. The access arrangements process was frequently described as 'bureaucratic', with particular criticisms of the required duplication of printing online records and of the online application form not being appropriate for all student awards (e.g. iGCSEs, Functional Skills): 
'The inclusion of support plans over the years that the student has been in the school, which includes advice to teachers, should be sufficient to demonstrate the normal way of working.' (R242, Assessor).

The impact of evidence collection demands and the incorporation of annual JCQ guidance changes were seen to vary according to the number of students requiring access arrangements in a particular year. Some respondents also highlighted both the complexity and ambiguity of JCQ's access arrangements guidance and schoolbased organisation relating to that:

'It is clear that even the exam boards are still finding their way. The JCQ guidelines need to be clearer as well - they can be confusing' (R83, SENCo)

'It is laborious but we have developed a system which works for us. However, part of it working depends on staff being experienced in what they need to do, and when staff leave, everything becomes difficult again for a while.' (R79, SENCo).

'As JCQ regulations change slightly every year, it means you can't start early enough and get prepared.' (R243, SENCo/ Assessor)

There were numerous indications that resource constraints were inhibiting both the process of identifying need and making an appropriate provision. Two respondents indicated knowledge of a more efficient access arrangements system in Scotland where there is greater executive delegation to schools:

'In very large schools [it's] not always possible to screen entire year groups as it would lead to even more exam arrangements. Sometimes we struggle to find spaces, or TAs [teaching assistants]'. (R62, SENCo)

'As the only (part time) Assessor at a large mixed comprehensive school I believe that only the most needy students are able to access assessment as well as a few students that are brought to my attention by staff. I would not have the time and the school does not have the resources to accommodate the needs of any more, though I believe many more would qualify if resources, time and in class support were available' (R221, Assessor)

'There is too much bureaucracy and top-down accountability - systems like in Scotland which allow for professional trust are much more effective and efficient' (R97, SENCo) 
Support for realistic and/ or additional staff and material resourcing from school leaders was, both directly and indirectly, identified by several respondents as essential to making the system of access arrangements manageable in school:

'The amount of work involved can no longer be carried out by the traditional staff roles. SLT need to support this by putting systems/staff in place to manage' (R94, Exams officer).

Even though prior communication with the school is required by JCQ access arrangements guidelines, a small minority of respondents felt that external assessor reports commissioned directly by parents could also affect manageability, and several respondents highlighted rising demand for access arrangements due to increased inclusion of students with SEN, as well as parental advocacy.

\section{Fairness of GCSE access arrangements}

Almost half of respondents (46\%) felt that the present system of access arrangements is fair, whilst just over half of respondents (51\%) expressed ambivalent or negative views about their fairness, focusing upon two areas:

- Eligibility criteria issued by JCQ

- Factors outside of the JCQ regulations, which may impinge upon the administration of access arrangements.

In respect of JCQ eligibility criteria some respondents questioned the 'translation' of 'normal way of working' to formal assessment situations from a variety of learning situations:

'Too much emphasis is placed on normal way of working. I accept that the learner needs to have used the computer etc. whilst studying but it does not reflect exam conditions. For example, students often word process their homework and work best on a computer but cannot always demonstrate this in the classroom' (R242, role not specified).

A wide variety of questions was raised about the validity and operation of specific tests and test cut-off scores for eligibility for access arrangements:

'I have just diagnosed a student of 'well above' average intelligence as dyslexic, this impacts on his ability to spell and organise his ideas effectively on paper. However, because his standardised assessment scores fall above 95 he cannot, through the online system get extra time, despite the fact that there is a significant discrepancy between these scores and his underlying ability.' (R204, Assessor). 
'In exam conditions, especially with a block of exams and long exams...they are more vulnerable to pressure which breaks down their resilience quite quickly'. (R253, Assessor).

'ASC [autistic spectrum condition] students sometimes confuse meanings of questions and small clarifications are needed... They rarely qualify for an OLM [oral language modifier] as their Reading Comp is usually $>69$. I feel they are unfairly disadvantaged.' (R231, Assessor).

'However for extra time re processing speeds, should those who fall under the banner of mental health issues have these evidenced by standardised scores too?' (R122, Support teacher).

'Some EHCP [Education, Health and Care Plan] students don't need extra time, bizarre that they automatically receive without assessment.' (R155, Assessor).

Fairness concerns unrelated to JCQ eligibility criteria often referenced the aforementioned human and material resource deficits for the administrative processes and provisions of access arrangements, particularly the effects of a lack of whole-cohort screening. Respondents also cited inequities created by differences in school capacities to devote resources to the processing and provisioning of access arrangements:

"I am well aware that many more students in this [independent] sector have access to access arrangements [examination access arrangements] than in the state sector' (R194, Assessor).

'There are children in Year 9 who cannot read and it is very much a lottery dependent on the staff and the finances of school as to what arrangements are put in place.' (R93, Assessor).

In respect of screening deficiencies, one respondent highlighted a greater role for students' active participation in identification:

'The school should talk about them to [students in] year 9, so they are able to refer themselves. Children are reluctant to use these as they feel it makes them feel different from their peers.' (R240, role unspecified).

Some respondents specifically mentioned unfairness stemming from differences between schools in access to, and expertise in, the use of assistive technologies within access arrangements. Whilst one respondent mentioned parental reluctance to support access arrangements on account of 'labelling', several respondents cited 'parental pressure'/ advocacy, know-how or ability to fund eligibility assessments as a factor which created unfairness in the system: 
'I think greater investment in technology in schools and the exam system could improve this. The exam system should reflect true life and the work environment.' (R94, Exams Officer).

'For medical/social emotional issues access to external help [is] very patchy and often dependent on parent ability to pay.' (R10, Assessor).

One respondent highlighted the benefit of a smaller examination room for those students experiencing examination stress, but was concerned that this was not always allowed as stresses additional to the examination itself had to be evidenced.

\section{Extension of GCSE access arrangements}

Almost two thirds of respondents (64\%) considered that extension of access arrangements would, notwithstanding resource constraints, be appropriate, whilst almost one quarter of respondents (22\%) considered that such extension would not be appropriate. (Though this latter figure may be an overestimate as a small number of respondents interpreted the question from their current adequate resource perspective). Six respondents indicated that extension would not be appropriate because access arrangements may serve to reduce student 'independence', with students coming to rely upon them. Furthermore, several responses seemed not to be framed from a student needs perspective, but from a resource-led or delimitation perspectives, including the notions of 'genuine need' and access arrangements as an 'award' (reminiscent of their former status as 'concessions'):

'there appear to be increasing numbers of pupils being awarded access arrangements and they can sometimes become over-reliant on this provision...I believe it is our role to give provision to those that really need it' (R191, Assessor).

'Screening at the start of each year/key stage using a variety of test might result in more access arrangements being awarded but not sure we should be actively "fishing" to award access arrangements' (R32, Assessor).

Whilst three respondents cited occasions of non-uptake of allowed access arrangements as a disincentive for any extension, more respondents acknowledged the benefits to student confidence of the very knowledge of the availability of a provision that might or might not be needed on the day of the examination. Several respondents highlighted the main barrier of financial constraints when contemplating any extension to current provisions, for example, in funding for the training and provision of staff to act as readers or oral language modifiers. 
Support for extension of access arrangements fell mainly into two, somewhat overlapping, categories:

- extension according to 'assessment need'

- extension through the development of technological assistance.

\section{Expansion according to assessment need}

In the context of aforementioned critical resource deficits, the principle that allowance of access arrangements should be 'needs-led' rather than 'resources-led' was frequently emphasised by those respondents in favour of extending access arrangements. Several respondents opined that GCSE examinations should be tests of the stipulated assessment objectives/ specification, rather than tests of 'testtaking skills' or 'examination technique'. Extension of reading assistance was the most commonly cited possible extension as some GCSE examinations were considered to have high reading fluency and comprehension demands, particularly multiple-choice examinations. Further to this, some respondents identified the benefits to some students of extra time, a quiet room in which they could verbalise as they worked through the examinations, or of an oral medium of presentation:

'Our current exams add an additional time element, which is not part of most specifications, e.g. being able to do maths quickly is not on the GCSE maths specification. Like in some other countries (e.g. Russia), I think exams times should be extended for all students to allow them to show what they know using as long or short a time as they need. Similarly, I don't see why all students shouldn't have access to a computer reader or word processor if that is their preferred way of working.' (R257, Assessor).

'There are very few occasions in life today where one needs to write under pressure in strictly timed conditions. If that were a pre-requisite of a place of work, they could screen using timed tests.' (R78, Assessor).

'Extend access arrangements to oral presentation as well as written, and allow everyone the choice. Writing penalises many pupils whether on paper/word processor.' (R27, Assessor).

\section{Expansion through technological assistance}

There were numerous indications of the possibility for expansion of access arrangements through the extended availability of technological assistance, including greater availability of laptops in GCSE examinations, reading pens, speech 
recognition (dictation) software and digital examination papers. This aspiration was often justified with reference to unmanageable levels of need for other forms of support requiring human assistance, e.g. reader, scribe.

Fully detailed analysis of findings relating to the use of assistive technologies within access arrangements is reported elsewhere (James, Hipkiss \& Woods, in preparation).

\section{Discussion and implications}

\section{Partnership with stakeholders in GCSE access arrangements}

Whilst some progress has been made with respect to perceived fairness of the system of administration of access arrangements for GCSE, it is still the case, as in 2007 , that the majority of those involved in some aspect of managing the arrangements does not consider the system to be equally fair to students. Furthermore, a sizeable majority continues to consider the system not to be reasonably manageable, and a majority still considers some extension of GCSE access arrangements to be appropriate. As in 2007, manageability is seen as hindered by JCQ regulations being changeable, unnecessarily complex and timeconsuming, though a new theme within this survey was the specific problem of timescale feasibility for evidence gathering in the further education sector, particularly under current policy of compulsory re-sits for English and Mathematics failed at high school (DfE, 2014a).

These findings are, however, perhaps not altogether surprising given that there still exist no mechanisms for the systematic gathering or use of, or response to, stakeholder views or satisfaction with GCSE access arrangements. The absence of effective service user engagement in this area appears anachronistic to a wide range of developments over the last 30 years of service user partnerships within health, education, care and commercial services (e.g. Barnes, Carpenter, \& Bailey, 2009; Goossen \& Austin, 2016; McKeown, Malihi-Shoja, \& Downe, 2010). In the absence of comprehensive and reliable data from the service user base, effective developments of the structure and processes of access arrangements through service user feedback is not possible. Therefore, a main implication of the findings of the present survey is that, in order to move towards the extolled best practice ideal (JCQ, 2004; Woods, 2007), a range of stakeholders, including young people, employers, and SENCos, should be regularly and systematically consulted and communicated to by Ofqual, with data being made available to the JCQ which oversees the detailed management of access arrangements. Through these processes, it would be possible 
to evaluate the feasibility of resolving or managing some of the dissatisfactions expressed within the present study.

\section{A school-based protocol for the management of GCSE access arrangements}

The present survey has highlighted the importance of an explicit and fit-for-purpose within-school/ college system for the effective management of GCSE access arrangements, to include realistic evaluation of resource demands, recognised in turn by the school's senior leadership team. Whilst the JCQ guidance can be seen to provide a framework, albeit not universally approved, for 'what' to do in relation to GCSE access arrangements, its dislocation from school contexts and stakeholders has left unaddressed the equally important question of 'how' to manage the organisation and delivery of them. Woods, Parkinson and Lewis (2010) identified the need for an in-school GCSE access arrangements protocol, and Hipkiss (2017) has described the development of such a protocol, highlighting both explicit staff roles and the recognition of staff resources needed for GCSE access arrangements. Notably, Hipkiss (2017) does not indicate a role for key school governors, such as the SEN governor and parent governor, though this may be useful to the effective and stable governance of a school's system for management of examination access arrangements.

In this research, the link between greater use of access arrangements and higher levels of resourcing, for example through the independent education sector, was confirmed (cf. Bateman, 2017). This suggests that concerns about rising levels of applications for access arrangements may be misplaced, as this is likely to represent schools and colleges better managing and resourcing the systems for identification of student assessment needs.

Against this background of resource deficits and compromised manageability, it is concerning that respondents often identified as negative the role of parental advocacy or autonomy in relation to their child's GCSE access arrangements. Woods (1998) also found that SENCos reported a broadly negative view of parental advocacy ('the pushy parent') in a context where the provision of GCSE access arrangements at school-level was resource-led and limited, rather than needs-led and flexibly provisioned. Correspondingly, Woods, Parkinson and Lewis (2010) identified parent reports of unsatisfactory engagement with school systems in relation to their child's GCSE access arrangements management or provision. In the present study, one respondent suggested improvements in communications between school, parents and examination boards in order to develop more constructive parent-school partnerships. Such developments may be most 
effectively and efficiently delivered through central co-ordination by appropriate agencies such as Ofqual or awarding bodies.

\section{Shared understanding about the purposes of access arrangements}

Even in the face of current manageability concerns, there continues to be a prevailing view by staff working in schools that GCSE access arrangements are not equally fair across candidates and that the use of such arrangements should be extended. Given official and media concerns about rising levels of GCSE access arrangement applications (Ofqual, 2016; Woolcock, 2017), this suggests a possible need to develop greater shared understanding between users of GCSE qualification (students, schools, employers, parents) and awarding bodies, about the purpose, place and necessary limitations of access arrangements within GCSE.

Particular criticism is levelled at the use of eligibility criteria relating to reading or processing test scores, or 'normal way of working', on account of their perceived validity in relation to student needs within assessment. Referencing relevance to the contemporary workplace, and citing alternative systems in other countries, several respondents called for more inclusive, and efficient, systems of access arrangements incorporating students' own 'preferred way of working' (cf also Peacey \& Peacey, 2007). A broader question was also raised about the current format of GCSE examinations including the available choice of assessment medium, with particular reference to extensions of oral and aural presentation and response methods, and the regular incorporation of a range of technological assistance (considered more fully elsewhere - James, Hipkiss \& Woods, submitted)

Dissonance between the provisions of the current system for GCSE access arrangements and stakeholder perceptions might arise from under-engagement with stakeholders. As highlighted above, however, it may also arise, in part at least, from a different balance of focus between 'disability' and the requirements of the Equality Act 2010 (HM Government, 2010), and an individual's SEN and the comprehensive detailing of special provisions associated with SEN legislation (DfE, 2015b), with the latter being the more practical concern in an educational context. Ofqual's existing Access Consultation Forum should develop its remit, using systematically gathered data as a basis for the development of shared understanding with all stakeholders about the purpose and effectiveness of access arrangements (Ofqual, 2017).

It should be noted, however, that the views of school-based practitioners on GCSE access arrangements were not entirely unequivocal, with a minority of respondents espousing benefits to dealing with attainment demonstration barriers akin perhaps

Author's version. Published as Woods, K., James, A., \& Hipkiss, A. (2018). Best practice in access 
to the development of 'examination technique', or supporting active delimitation of access arrangements to only the most significant needs.

\section{Limitations}

The present study is limited by its self-selecting respondent sample. In this context, however, self-selecting participants were likely to be relatively well informed, as borne out by the extensively detailed information provided by many respondents, and so the findings of this study are likely to be close to a 'best case scenario'. A further limitation relates to the survey questionnaire's inclusion of some multi-part questions, giving rise to a small proportion of ambiguous responses to parts of a question.

\section{References}


American Psychological Association (APA) (2006). Evidence Based Practice in Psychology, American Psychologist, May-June, 271-285.

Barnes, D., Carpenter, J., \& Bailey, D. (2009). Partnerships with service users in interprofessional education education for community mental health: A case study. Journal of Interprofessional Care, 14(2), 189-200.

Bateman, T. (2017). Independent schools gain extra time for exams. Retrieved from: http://www.bbc.co.uk/news/education-38923034

Biesta, G. (2007). Why what works won't work: Evidence-based practice and the democratic deficit in educational research. Educational Theory, 57(1), 1-22.

British Psychological Society (BPS) (2014). Code of human research ethics. Leicester: British Psychological Society.

Cohen, L. , Manion, L., \& Morrison, K. (2011) Research methods in education (7 $7^{\text {th }}$ edition). London: Routledge.

DeLuca, C., \& Johnson, S. (2017). Developing assessment capable teachers in this age of accountability. Assessment in Education: Principles, Policy and Practice, 24(2), 121126.

Department for Education (DfE) (2014a). Assessment curriculum and qualifications: Research priorities and questions. Nottingham: HMSO.

Department for Education (DfE) (2014b) Children and Families Act 2014. Nottingham: HMSO.

Department for Education (DfE) (2015a). New reforms to raise standards and improve behaviour. Retrieved from: https://www.gov.uk/government/news/newreforms-to-raise-standards-and-improve-behaviour

DfE (2015b). Special educational needs and disability code of practice: 0 to 25 years. London: Department for Education.

DfE (2017) Revised GCSE and equivalent results in England: 2015 to 2016. London: Department for Education.

Flitcroft, D., Woods, K., \& Putwain (2017). Developing practice in preparing students for high-stakes examinations in English and Mathematics. Educational and Child Psychology, 34(3), 7-19. 
Gillham, B. (2007). Developing a questionnaire. London: Continuum International Publishing Group.

Goossen, C., \& Austin, M.J. (2016). Service user involvement in UK social service agencies and social work. Journal of Social Work Education, 53(1), 37-51.

HM Government (2010). Equality Act 2010: Guidance. London: HM Government (Office for Disability Issues).

Hipkiss, A. (2017). Development of a model for the identification and management of student assessment needs: utilising student voice and student experience. The University of Manchester: Presentation to ESRC project advisory group (available from author).

Hipkiss, A., \& Robertson, C. (2016). The impact of access arrangements organisation on Special Educational Needs Co-ordinators (SENCos). The University of Manchester: Unpublished report (available from first author).

Hsieh, H-F., \& Shannon, S.E. (2005). Three approaches to qualitative content analysis. Qualitative Health Research, 15(9), 1277-1288.

Isaacs, T. (2010). Educational assessment in England. Assessment in Education: Principles, Policy \& Practice, 17(3), 315-334.

James, A., Hipkiss, A., \& Woods, K. (In preparation). Teacher views on the use and scope of assistive technologies for GCSE examinations. British Journal of Educational Technology.

Joint Council for Qualifications (JCQ) (2004). Access arrangements and special consideration: Regulations and guidance relating to candidates who are eligible for adjustments in examinations. London: JCQ.

Joint Council for Qualifications (JCQ) (2008). Access arrangements, reasonable adjustments and special consideration: General and vocational qualifications with effect from

Joint Council for Qualifications (JCQ) (2017). Adjustments for candidates with disabilities and learning difficulties: Access arrangements and reasonable adjustments with effect from 1 September 2017 to 31 August 2018. London: JCQ.

McKeown, M., Malihi-Shoja, L., \& Downe, S. (2010). Service user and carer involvement in education for health and social care. Chichester: Wiley. 
Ofqual (2016). Statistical Release. Access arrangements for GCSE and A level: 20152016 academic year. London: Qfqual.

Ofqual (2017). Ofqual Corporate Plan 2017-2020. London: Ofqual.

Peacey, N., \& Peacey, L. (2007). Minimising bias in assessment for students with special educational needs and disabled students: Reasonable adjustments in written national curriculum tests at key stage 2 and key stage 3. London: Qualifiation and Curriculum Authority (QCA).

Stobart, G. (2008). Testing times: The uses and abuses of assessment. Abingdon, Oxon: Routledge.

Woods, K. (1998). School processes in selection for GCSE special examination arrangements, Educational Psychology in Practice, 14 (3), 59-67.

Woods, K. (2007). Access to the General Certificate of Secondary Education (GCSE) examinations for students with special educational needs: what is best practice?, British Journal of Special Education, 34(2), 89-95.

Woods, K., McCaldin, T., Hipkiss, A., Tyrell, B., \& Dawes, M. (Submitted). Making the link between children's rights and systems of educational assessment. Oxford Review of Education.

Woods, K., Lewis, S., \& Parkinson, G. (2010). Investigating access to educational assessment for students with disabilities, School Psychology International, 31(1), 2141.

Woolcock, N. (2017). Middle-class parents drive surge in students given extra time for exams. Retrieved from: https://www.thetimes.co.uk/article/middle-classparents-drive-surge-in-students-given-extra-time-for-exams-k5nh677ja

\footnotetext{
i Progress 8 is a type of value added measure, introduced in 2016 by the English Government's Department for Education, by which students' GCSE outcomes at age 16 are compared to the achievements of other students with similar prior attainment at age 11 .
} 